\title{
Chronic Meningococcemia
}

\author{
Rita Veiga Ferraz, Filipa Ceia, Raquel Duro, Ana Cláudia Carvalho, Paulo Andrade, \\ Susana Silva, António Sarmento \\ Infectious Diseases Department, Instituto de Inovação e Investigação em Saúde (I3S), Grupo de I\&D em \\ Nefrologia e Doenças Infeciosas, Instituto Nacional de Engenharia Biomédica (INEB), Centro Hospitalar de São \\ João, Porto, Portugal \\ Email: ferrazrit@gmail.com, fsfceia@gmail.com, raquel.duro@gmail.com, anaclaudiacarvalho@gmail.com, \\ andrade.pf@gmail.com, susanamaiasilva@gmail.com, antoniosarmento55@sapo.pt
}

Received 5 June 2016; accepted 30 July 2016; published 2 August 2016

Copyright (C) 2016 by authors and Scientific Research Publishing Inc. This work is licensed under the Creative Commons Attribution International License (CC BY). http://creativecommons.org/licenses/by/4.0/

(c) (i) Open Access

\begin{abstract}
Neisseria meningitidis is a gram-negative diplococcus which causes invasive disease. The most frequent clinical manifestations caused by infection with this pathogen, are meningitis and fulminant septic shock. More benign forms with a sub-acute or chronic pattern are rare but still can evolve to meningitis and have a fatal outcome. This entity is difficult to recognize, as febrile erythema with otherwise good general condition may simulate other diseases, namely viral infections. The authors reported the cases of two healthy young adults who were misdiagnosed with a viral infection. What they really had was a meningococcemia without meningitis presenting with mild and unspecific symptoms, such as, intermittent fever and a discrete macular rash. We pretend to highlight this rare, unspecific and heterogeneous symptoms which can provoke negligence of a potentially life threatening condition.
\end{abstract}

\section{Keywords}

Neisseria meningitidis, Meningococcal Disease, Chronic Meningococemia

\section{Introduction}

Neisseria meningitidis is a gram-negative diplococcus, pathogenic only in humans, that colonizes the nasopharynx asymptomatically in up to $5 \%$ - $10 \%$ of the adult population and is transmitted from person to person through respiratory secretions [1]. The meningococcal invasive disease is associated only with six serogroups classified on the basis of polysaccharide capsule (A, B, C, W-135, X, and Y). Serogroups B and C cause disease predominantly in Europe and the Americas [2] and the estimated incidence in Europe is of 1.01 per 100,000 population [3] [4]. The most frequent clinical manifestations are meningitis and fulminant septic shock [5]. 
Chronic meningococcemia is a rare form of meningococcal sepsis without meningitis, characterized by recurrent fever for at least 1 week, arthralgia and skin eruption [6].

The authors present two cases of meningococcemia without meningitis and with mild and unspecific symptoms which could have lead to serious events if not recognized and treated promptly.

We consider these reports which demonstrate, exactly, how we misdiagnosed a meningococcal infection, of major importance, to highlight the physicians of the possibility of unspecific presentation of meningococcal disease, which can have a high mortality rate.

\section{Case Reports}

\subsection{Case 1}

A 20 year-old Caucasian man presented to the ER on the $20^{\text {th }}$ of March 2015 complaining of fever, fatigue and mild headache for the last 48 hours, associated with the development of a macular rash involving the palms and plants in the previous 12 hours.

His previous medical history was unremarkable. He worked as a physical therapist with no contact with children. He reported a holiday stay in Nice (France) 2 months before and also unprotected sexual behaviour. There was no other relevant epidemiological data.

In the ER, his temperature was $36^{\circ} \mathrm{C}$, pulse was regular, 70 beats $/ \mathrm{min}$, blood pressure was of $120 / 70 \mathrm{mmHg}$, respiratory rate of $18 \mathrm{rate} / \mathrm{min}$. There was a diffuse macular rash with palmar and plantar involvement but the remaining physical examination was normal. Initial laboratory data revealed leukocytosis (white blood cell (WBC) count of $22.870 \times 10^{9} / \mathrm{L}$ with $20.700 \times 10^{9} / \mathrm{L}$ neutrophils), elevated C-reactive protein $(271 \mathrm{mg} / \mathrm{L})$, normal renal and hepatic panels and negative urinalysis. HIV check and Monospot test were negative. Blood cultures were collected.

The patient was discharged on the same day, with oral doxycycline, assuming a zoonosis. He improved over the following days, with sustained apyrexia and resolution of the rash.

Seven days after the initial observation (on the $27^{\text {th }}$ of March) we were aware of serotype B Neisseria meningitidis isolation in the blood cultures. The patient was contacted for hospital admission; he was asymptomatic and his physical examination was normal. On hospital admission new blood cultures were collected, and no growth was detected. He started ceftriaxone $2 \mathrm{~g}$ bid with good outcome and was discharged after having completed 7 days of therapy. During follow-up no events were reported.

\subsection{Case 2}

A 24 year-old Caucasian, previously healthy woman, presented to the emergency room (ER), on the $2^{\text {nd }}$ of $A u-$ gust 2015, with a history of sudden onset of fever (maximum $39^{\circ} \mathrm{C}$ ), polyarthralgia, initially localized to the ankle and subsequently to the knee and wrist with proximally 6 hours duration, and a mild maculopapular rash localized mainly to the arms and palms noticed in the previous hour.

On what concerns to the epidemiological context, the patient was a psychologist, who worked with children, and denied any relevant contact with animals or rural areas, non-pasteurized products consumption or history of travel.

On examination at the ER, pulse was regular, 70 beats/min, blood pressure was of 100/60 mmHg, respiratory rate of 16 rate/min, auricular temperature of $36,5^{\mathrm{a}} \mathrm{C}$, and a macular rash mainly localized to the limbs was evident. Oropharynge showed no exudates, no arthritis was present and the reminder of physical examination was unremarkable.

Laboratory exams revealed a haemoglobin of $11.8 \mathrm{~g} / \mathrm{dL}$, leucocytes of $9.000 \times 10^{9} / \mathrm{L}$, neutrophilia of $82 \%$, platelets of $276.000 \times 10^{9} / \mathrm{L}$. C-reactive protein (CRP) of $59 \mathrm{mg} / \mathrm{L}$. Liver enzymes and renal function were within normal range.

A viral infection was presumed, but still, blood cultures were collected. HIV test and Treponema pallidum particle agglutination assay (TPPA) were negative. Patient was discharged with symptomatic treatment and a re-evaluation planned three days after.

On the second observation ( $5^{\text {th }}$ of August), she reported no new episodes of fever and symptoms were improving. One of the two blood cultures was positive, no agent was yet identified (contamination was suspected) and again she was discharged, planning re-evaluation within one week. 
Unexpectedly, 24 hours after (6 $6^{\text {th }}$ of August) the fever and arthralgia re-appeared and a slight petechial rash started to emerge in the limbs. Patient was admitted to the Infectious Diseases Department and ceftriaxone (2 g bid) was promptly initiated, assuming the possibility of meningococcemia. The rash progressed and petechial lesions extended to the limbs including palms and soles and also tongue (Figures 1-3).

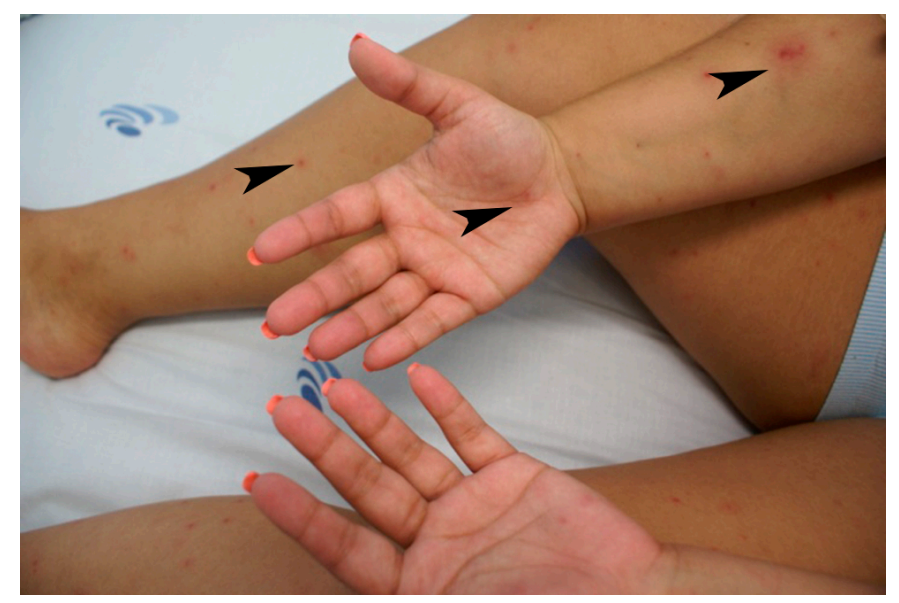

Figure 1. Case 2, petechial lesions localized to the arms, palms and legs.

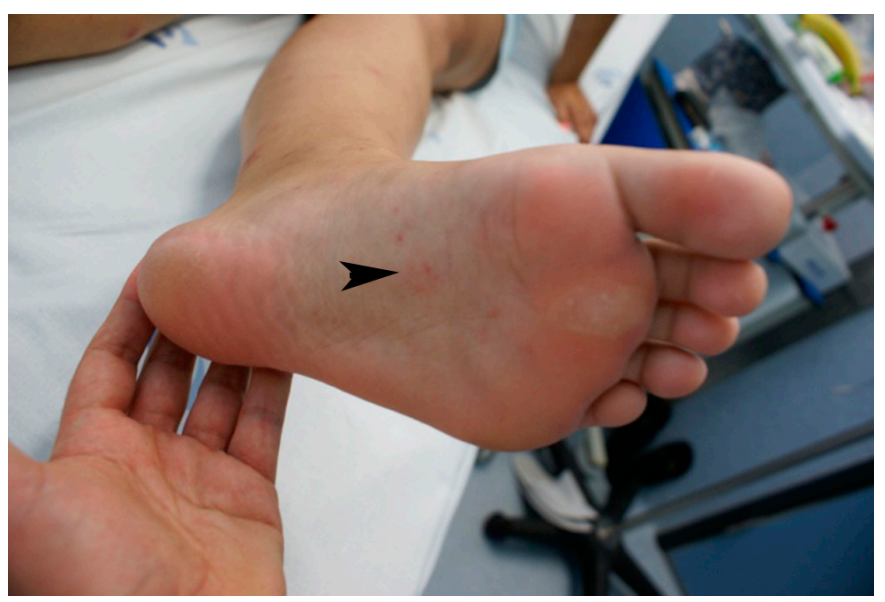

Figure 2. Case 2, petechial lesions localized to the soles.

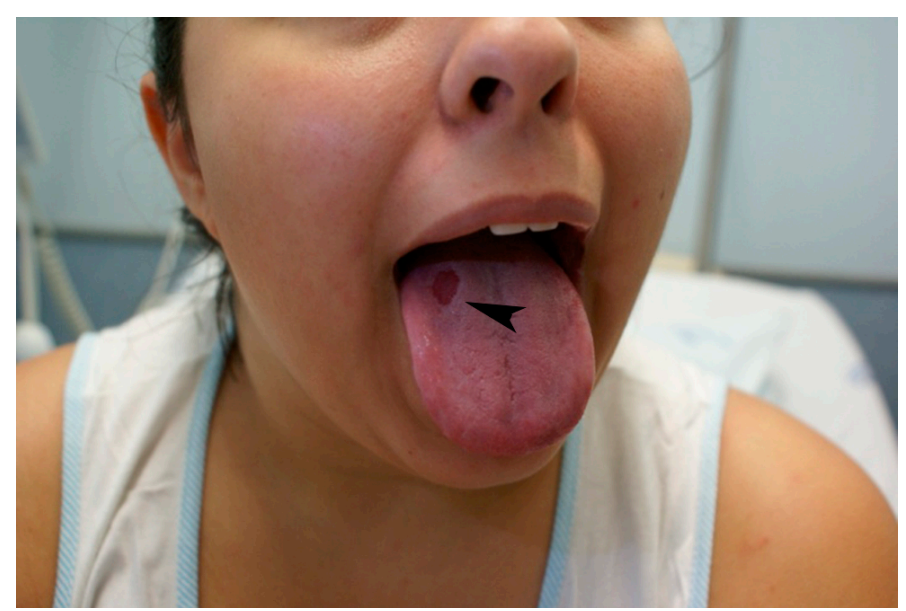

Figure 3. Case 2, petechial lesion in the tongue. 
Blood cultures [2] performed on the first ER observation ( $2^{\text {nd }}$ of August) yielded Neisseria meningitidis, serotype was not identified.

Forty-eight hours after, the patient gradually improved, rash started to disappear and she became afebrile. She was discharged 7 days later completely recovered.

During follow-up no events were reported.

\section{Discussion}

The most commonly recognized presentation of meningococcal disease, globally, accounting for $80 \%$ to $85 \%$ of all reported cases, is Meningococcal meningitis (in half of these cases sepsis is also present concomitantly). The remaining $15 \%$ - $20 \%$ of cases are most commonly bloodstream infections without meningitis [2]. This condition is characterized by abrupt onset of fever and a petechial or purpuric rash, often associated with hypotension, shock, acute adrenal hemorrhage, and multiorgan failure [10]. Alongside the classic acute forms of the disease, there are rare chronic (benign) forms, defined as a meningococcal sepsis of at least one week duration without meningeal symptoms and characterized by a prolonged clinical course with intermittent fever, rash and migratory arthralgia in healthy individuals [7]. Chronic meningococcemia may be self-limiting, but meningitis and death can occur as a late complication [8].

The cardinal feature of a meningococcal sepsis is the purpuric rash, which is a late sign of the disease, usually begins as an erythema, but later evolves to petechiae and purpura due to dermal microvascular thrombosis and perivascular haemorrhage [9]. Despite the existence of skin rash, these atypical forms of subacute or chronic meningococcemia are difficult to recognize, mostly in erythema stage as other diseases may simulate an identical picture, namely viral illnesses.

Considering our patients, although the duration of the symptoms was slightly shorter than one week, the clinical presentation was extremely benign (namely, the intermittent fever, the migratory arthralgia and the rash with otherwise good general condition), characteristic of chronic meningococcemia.

The gold standard for the diagnosis of meningococcal disease remains the culture by isolation of N. meningitidis from a normally sterile site. The diagnosis is challenging as bacterial cultures are frequently negative in the initial stages of the illness. Polimerase Chain Reaction (PCR) is useful for detection of $N$. meningitidis from clinical samples in which the organism could not be detected by culture methods, such as when a patient has been treated with antibiotics before obtaining a clinical specimen for culture [10], but it was not performed in our patients as the culture was already positive.

The reason why these less severe forms of disease occur remains unknown. Susceptibility of the host and the virulence of the Meningococcus have been referred as possible explanations. $N$. meningitidis of serogroup B seems to be more often involved than other serogroups. Some authors state that the relatively reduced virulence of serogroup B may partly explain the chronicity of clinical signs and the reduced inflammatory response [11] [12].

Once $N$. meningitidis infection has been confirmed, penicillin alone is the recommended treatment, although a $3^{\text {rd }}$ generation cephalosporin may be considered [10] due to the greater convenience and simplicity of the regimen.

Hopefully, our cases were recognized early in the course of the disease, enabling timely treatment that led to a favorable outcome.

\section{Conclusions}

In conclusion, meningococcal infection should be always included as a differential diagnosis of fever, especially in the presence of skin rash or petechiae, even when there are no signs of meningitis.

Reporting these particular cases, the authors pretend to highlight the unspecific and heterogeneous symptoms with which meningococcal disease can present, leading to misdiagnosis of a potentially life threatening condition.

\section{Acknowledgements}

To Candida Abreu for her help reviewing this paper. 


\section{References}

[1] Coureuil, M., et al. (2013) Pathogenesis of Meningococcemia. Cold Spring Harbor Perspectives in Medicine, 3, a012393.

[2] Sabatini, C., Bosis, S., Semino, M., Senatore, L., Principi, N. and Esposito, S. (2012) Clinical Presentation of Meningococcal Disease in Childhood. Journal of Preventive Medicine and Hygiene, 56-119.

[3] Thompson, M.J., et al. (2006) Clinical Recognition of Meningococcal Disease in Children and Adolescents. Lancet, 367, 397-403. http://dx.doi.org/10.1016/S0140-6736(06)67932-4

[4] Tan, L.K., Carlone, G.M. and Borrow, R. (2010) Advances in the Development of Vaccines against Neisseria meningitidis. New England Journal of Medicine, 362, 1511-1520. http://dx.doi.org/10.1056/NEJMra0906357

[5] Poizeau, F., et al. (2015) Meningococcémie sans méningite: À propos de deux observations. Revue de Médecine Interne.

[6] Dupin, N., et al. (2012) Chronic Meningococcemia Cutaneous Lesions Involve Meningococcal Perivascular Invasion through the Remodeling of Endothelial Barriers. CID, 54, 1162-1165. http://dx.doi.org/10.1093/cid/cis120

[7] Kernéis, S., Mahé, E., Heym, B., Tardy, V.S., Bourgeois, F. and Hanslik, T. (2009) Chronic Meningococcemia in a 16Year-Old Boy: A Case Report. Cases Journal, 2, 7103. http://dx.doi.org/10.4076/1757-1626-2-7103

[8] Harwood, C.A., Stevens, J.C., Orton, D., Bull, R.C., Paige, D., Lessing, M.P., Mortimer, P.S., Marsden, R.A. and Cerio, R. (2005) Chronic Meningococcaemia: A Forgotten Meningococcal Disease. British Journal of Dermatology, 153, 669-671. http://dx.doi.org/10.1111/j.1365-2133.2005.06771.x

[9] Hazelzet, J.A. (2005) Diagnosing Meningococcemia as a Cause of Sepsis. Pediatric Critical Care Medicine, 6, S50S54. http://dx.doi.org/10.1097/01.PCC.0000161947.57506.D6

[10] (2015) Meningococcal Disease. Centers for Disease Control and Prevention Epidemiology and Prevention of Vaccine-Preventable Diseases, 13th Edition.

[11] Fasano, M.B., Sullivan, K., Ibsen, L. and Winkelstein, J.A. (1993) Chronic Meningococcemia in a Child with a Deficiency of the Sixth Component of Complement. Pediatric Allergy and Immunology, 4, 214-216. http://dx.doi.org/10.1111/j.1399-3038.1993.tb00094.x

[12] Boos, C., Daneshvar, C., Hinton, A. and Dawes, M. (2004) An Unusual Case of Chronic Meningitis. BMC Family Practice, 5, 21. http://dx.doi.org/10.1186/1471-2296-5-21

\section{Submit or recommend next manuscript to SCIRP and we will provide best service for you:}

Accepting pre-submission inquiries through Email, Facebook, LinkedIn, Twitter, etc.

A wide selection of journals (inclusive of 9 subjects, more than 200 journals)

Providing 24-hour high-quality service

User-friendly online submission system

Fair and swift peer-review system

Efficient typesetting and proofreading procedure

Display of the result of downloads and visits, as well as the number of cited articles

Maximum dissemination of your research work

Submit your manuscript at: http://papersubmission.scirp.org/ 